\title{
LOS HUMEDALES EN LA EVALUACIÓN DE IMPACTO AMBIENTAL DEL PROPUESTO AEROPUERTO DE CHINCHERO
}

\author{
Carlos Antonio Martin Soria Dall'Orso \\ Consultor Internacional
}

\begin{abstract}
Resumen: Este articulo revisa, a la luz del marco legal aplicable, algunos aspectos del Estudio de Impacto Ambiental (en adelante el EIA) de la propuesta de construcción y operación de un aeropuerto en Chinchero (ACON \& Kuntur Wasi 2015), relativos al análisis del impacto potencial de dicha obra sobre el sistema de humedales altoandinos con alto valor natural, cultural y socioeconómico.

Primero se presentan detalles básicos del distrito y pueblo de Chinchero, del propuesto aeropuerto de Chinchero y de los humedales. A seguir, se revisa la caracterización del EIA de los posibles impactos ambientales sobre el recurso agua. Luego se presentan los componentes del sistema de humedales altoandinos de Chinchero. Con este contexto se analiza la caracterización de los impactos ambientales en el EIA y qué elementos debieron constar en este, y con ello se revisa el marco legal aplicable para formular algunas conclusiones.

Las deficiencias de la línea base y las ausencias evidencian la pobre revisión del documento y del área de estudio durante el proceso de certificación y durante la fiscalización ambiental. La caracterización de los impactos ambientales sobre el sistema hídrico de humedales altoandinos de Chinchero es una grave ausencia. Las conclusiones permiten identificar acciones que las autoridades del sistema de gestión ambiental deben tomar para corregir las insuficiencias y poder evaluar de manera certera el impacto ambiental de la obra sobre el sistema hídrico de humedales altoandinos.
\end{abstract}

Palabras clave: ambiente, derecho ambiental, evaluación ambiental, humedales, Perú. 


\title{
Wetlands in the Environmental Assessment of the Proposed Chinchero Airport
}

\begin{abstract}
The article reviews an environmental assessment of a proposed airport in an area of high Andean wetlands with high natural, cultural and socioeconomic value. Besides a deficient base line, this paper proposes that a poor environmental certification and enforcement process allowed that the characterization of the environmental impacts of the proposed airport did not consider that the Chinchero high Andean wetlands are to be affected. The author reviews the applicable legal framework and concludes identifying actions that the authorities of the environmental management system must take to correct the insufficiencies and to be able to accurately assess the environmental impact of the proposed airport over the high Andean wetlands.
\end{abstract}

Keywords: Environment, Environmental Assessment, Environmental Law, Peru, Wetlands.

\section{Carlos Antonio Martin Soria Dall'Orso}

Abogado por la Pontificia Universidad Católica del Perú, Maestro en Ciencias Sociales con mención en Estudios Amazónicos por la Facultad Latinoamericana de Ciencias Sociales, Sede Ecuador, y Ph. D. por La Universidad Flinders de Australia del Sur. Docente universitario, investigador académico y especialista en asuntos amazónicos y de desarrollo sostenible con tres décadas de experiencia en el Perú y el extranjero. Se ha desempeñado profesional y académicamente en Perú, Ecuador, Australia, Estados Unidos de América, Chile y Mozambique. Es co-autor del Código del Medio Ambiente y los Recursos Naturales del Perú, así como de la Ley General del Ambiente, entre otras normas. Ha sido Secretario General del Servicio Nacional de Áreas Naturales Protegidas por el Estado, del Ministerio del Ambiente. Ha sido consultor con la Organización del Tratado de Cooperación Amazónica, con FAO, con Helvetas-CONDESAN, con USAID, del Centro Internacional para la Investigación Forestal CIFOR, entre otros.

Correo electrónico: carlosantoniomartin@gmail.com 


\section{El distrito de Chinchero}

El distrito de Chinchero, ubicado en gran parte de la meseta Chinchero-Maras, en la provincia de Urubamba, tiene una superficie de $94.57 \mathrm{~km}^{2}$ o $9.457 \mathrm{ha}$. Chinchero conecta al Cusco con los distritos de Maras, Urubamba, Ollantaytambo y Machupicchu, entre otros. El clima es semiseco, con una precipitación promedio de $750 \mathrm{~mm} /$ año; con lluvias entre diciembre y marzo, cuando el aire húmedo proveniente de la Amazonía ingresa al altiplano y se descarga en forma de lluvias convectivas (Grace 1983 citado por Municipalidad Distrital de Chinchero 2016: 43). La temperatura varía entre $8,4^{\circ} \mathrm{C}$ (junio a julio) hasta $14,8{ }^{\circ} \mathrm{C}$ (enero a marzo), con una máxima de 22 ${ }^{\circ} \mathrm{C}$ y una mínima de $-5^{\circ} \mathrm{C}$. La humedad relativa promedio máxima es $82,8 \%$ (enero) y la mínima $66,8 \%$ (agosto). Las estaciones meteorológicas Pisac y Anta-Ancachuro del Senamhi registran que la precipitación mensual varió de 198,1 mm (febrero) a 4,6 mm (mayo) en el periodo 2004-2013, con la mayor precipitación total en enero de 2010 (289,0 mm). Los días de precipitaciones igual o superior a 1 milímetro son 81 . El distrito tiene 2 centros poblados urbanos, Chinchero y Umasbamba, y 43 centros poblados rurales (Municipalidad Distrital de Chinchero 2016: 43).

Chinchero se encuentra situado en un punto alto que domina el Valle Sagrado de los Incas. El distrito de Chinchero, ubicado en la cabecera de la cuenca del río Urubamba, tiene 7 quebradas (Marcuyohuayjo, Sayocachi, Huaynanca, Quehuarhuayco, Sayac Qagahuayco, Cusihuayco, Cedrohuayjo), el río Ccorimarca y 27 lagunas entre mayores y menores, identificándose 2 microcuencas de drenaje: la de la laguna Huaypo Yanacona y la microcuenca Piuray-Corimarca. Tanto la meseta de Chinchero como los valles circundantes están poblados de andenes y canales.

Este sistema hídrico abastece de agua a Chinchero y las comunidades, pero también a la Ciudad del Cusco y los distritos de Urubamba y Anta. Los canales y manantiales abastecen de agua para su consumo a las comunidades ${ }^{1} \mathrm{y}$ para las áreas de riego,

«[...] un significativo 20,1\% (447) de viviendas se abastecen del río, acequias, manantiales o similares». (Municipalidad Distrital de Chinchero 2016: 39) «De acuerdo con la información proporcionada por OMSABAR, la zona rural de la Microcuenca Piuray, cuenta con fuentes de agua que les permiten tener su propio sistema de abastecimiento 
mientras que una prodigiosa naturaleza mantiene un fértil ciclo del agua que garantiza el abastecimiento anual. La riqueza natural de este sistema hídrico es un hábitat de flora y fauna altoandina, pero también una base importante para la agrobiodiversidad, que revela la sofisticación de la cultura inca al aprovechar las virtudes del espacio natural para construir una sociedad que se autoabastece y provee excedentes al intercambio.

La capital distrital, el pueblo de Chinchero, de origen inca, se ubica a $28 \mathrm{~km}$ de la ciudad del Cusco, a $3.780 \mathrm{~m} \mathrm{s.} \mathrm{n.} \mathrm{m,} \mathrm{flanqueada} \mathrm{por} \mathrm{grandes}$ nevados, como el Salkantay, Verónica y Soray, y por las lagunas Huaypo y Piuray. El poblado se encuentra en el centro de las tres principales comunidades campesinas: Yanacona, Ayllopongo y Cuper, en la ladera del cerro Antaccasa.

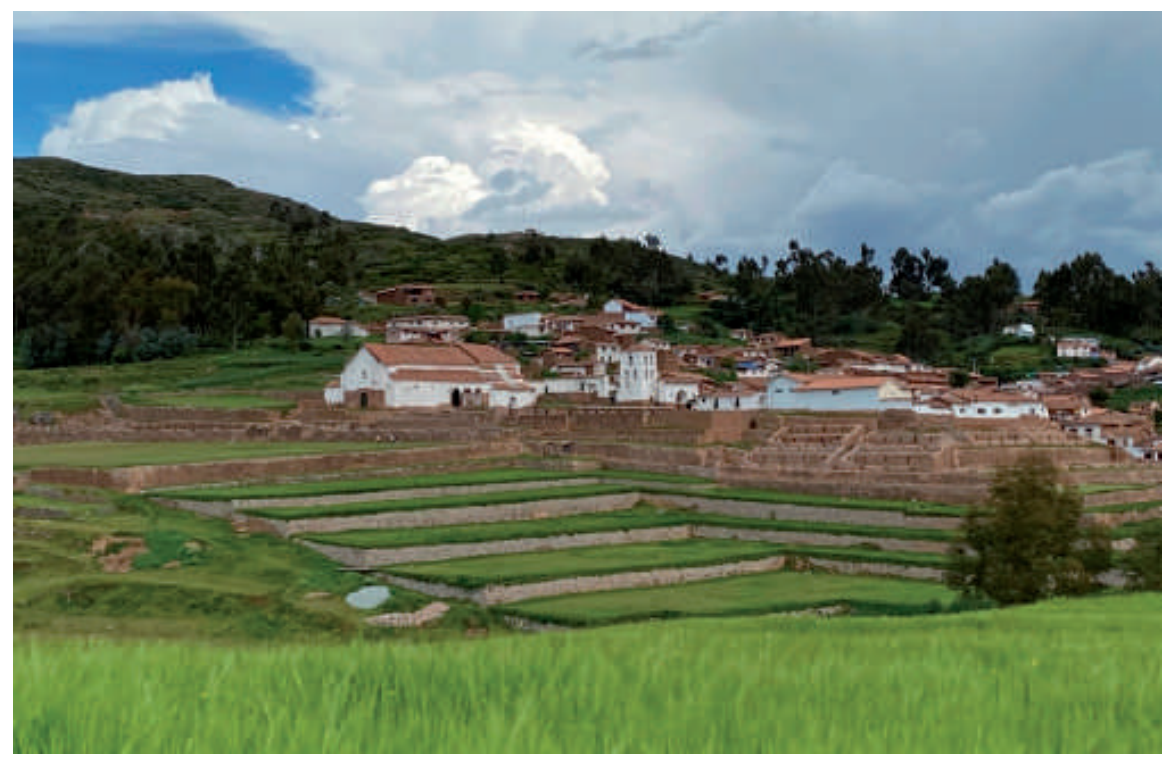

Imagen 1. Vista del palacio del Inca Túpac Yupanqui en Chinchero. Fuente: Soria (2020).

El aspecto más resaltante del pueblo de Chinchero es la presencia del palacio del Inca Tupac Yupanqui y el templo de Nuestra Señora de Monserrat, administrado por el pueblo y las comunidades, o ayllus, de Chinchero que explotan tierras para apoyar a la manutención del templo. El polígono del propuesto aeropuerto de Chinchero se superpone a parte de las tierras de la comunidad campesina Ayllopongo, destinadas al servicio del templo. La vida

de agua potable las 24 horas del día, atendiendo a 12 comunidades y/o sectores organizados en 12 JASS; para el año 2016, el 76,8\% (928 familias) cuentan con el servicio y el 23,2\% (281 familias) no cuentan con el servicio». (2016:41) 
de doce ayllus (sistema de organización territorial incaico) o comunidades campesinas indígenas andinas, dedicadas al cultivo de especies nativas, la ganadería y algo de turismo local, marcan el ritmo de la convivencia sociedad-naturaleza en el distrito de Chinchero.

Chinchero es un pueblo y un conjunto arqueológico que muestra la simbiosis entre el espacio construido por el hombre aprovechando la geografía, configurando así un patrimonio natural y cultural. Los andenes y terrazas son la evidencia de que estamos en lo que es un centro de producción y adaptación de especies y producción agrícola que ya existía en el periodo inca, se adaptó y sobrevivió en el periodo colonial y hoy continúa. La producción agrícola de Chinchero y sus comunidades son la base de la alimentación y la medicina tradicional de esta población. Esta producción, además, se intercambia con otras comunidades de los distritos aledaños del conjunto del Valle Sagrado.

La zona monumental de Chinchero fue declarada patrimonio histórico (resolución suprema 2900-1972-EDU). Luego, el Parque Arqueológico de Chinchero fue declarado como Patrimonio Cultural de la Nación (resolución directoral nacional 405-2002-INC). Finalmente, la declaración de Patrimonio Cultural de la Nación del Valle Sagrado de los Incas (resolución directoral nacional 988-2006-INC) contiene a los parques arqueológicos Pisac, Chinchero y Ollantaytambo, y otros 350 sitios arqueológicos.

\section{El proyecto de aeropuerto}

El polígono del propuesto aeropuerto de Chinchero ocupa ${ }^{2} 499,3$ ha en los distritos de Chinchero y Huayllabamba, provincia de Urubamba, departamento de Cusco, entre los 3.714 y 3.731 m s. n. m. El aeropuerto se emplazaría entre el nevado Pumahuanca y la cordillera nevada del Chicón, que llegan a más de 5.300 m s. n. m.

El área del polígono está conformada por unos campos, más o menos en planicie, en los que se localizaban áreas agrícolas pertenecientes a las comunidades campesinas Ayllopongo y Yanacona. Desde 2012, el Ministerio de Transportes y Comunicaciones (MTC) adquirió estas tierras, pagando precios exorbitantes a las comunidades campesinas. En 2017, se pagó USD 250.000 por 1 ha (La República 2017), cuando en Idaho, Estados Unidos de América, la tierra agrícola más cara no excede los USD 12.875 (Capital Press 2017) y en el Reino Unido de la Gran Bretaña la tierra agrícola más cara se vendió en Devon del Este a USD 43.400 la hectárea (Dodds 2018). 
El polígono del propuesto aeropuerto de Chinchero se ubica en la cuenca del Urubamba, en la microcuenca del rio Corimarca. Este ecosistema contiene lagunas y bofedales altoandinos. Chinchero tiene ambos, es decir, lagunas y bofedales, como es el caso de las lagunas Huaypo y Piuray, y los pequeños bofedales en la misma microcuenca de la laguna de Piuray ${ }^{3}$. Este tipo de humedal se puede encontrar en variado número alimentando a humedales más grandes, como es también el caso del sistema de humedales de Chinchero. El aeropuerto estará localizado entre las lagunas Huaypo y Piuray y se abastecerá de agua de la napa freática mediante bombeo (Novoa Arévalo 2003: 39-41).

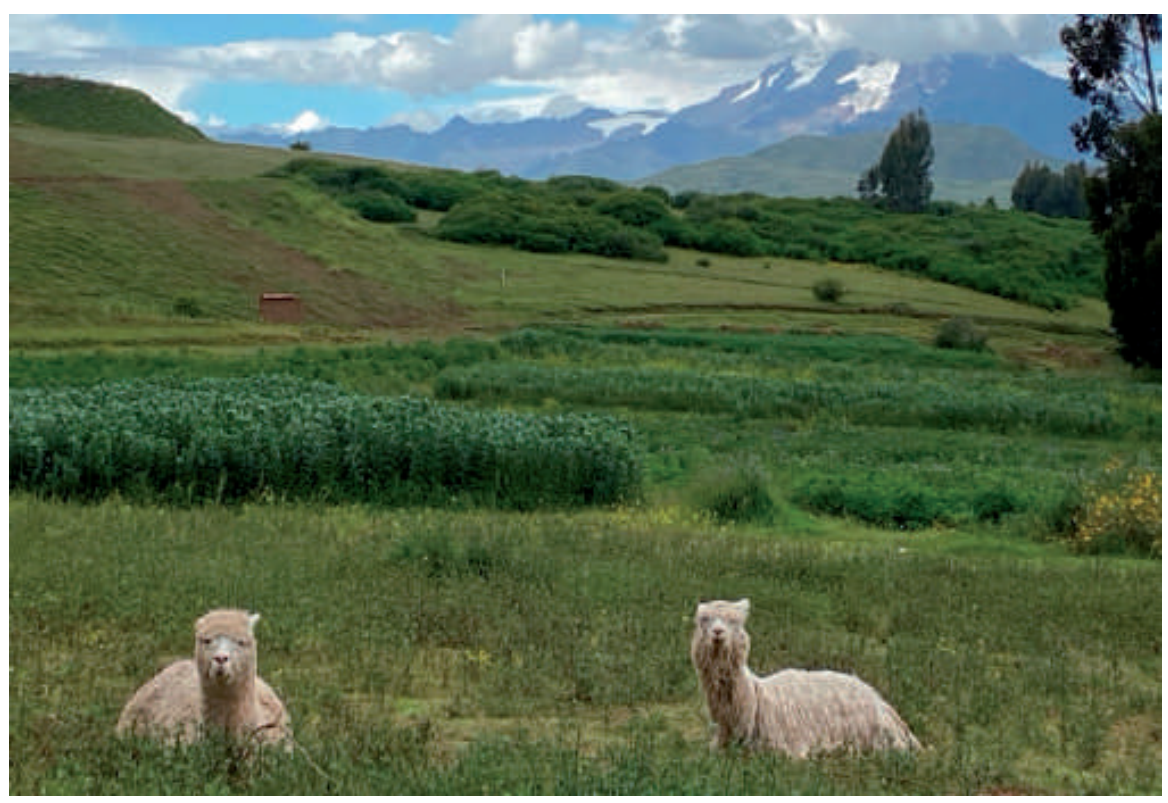

Imagen 2. Alpacas, cultivos y nevado en el paisaje de Chinchero. Fuente: Soria (2020).

\section{El propuesto aeropuerto de Chinchero y los humedales}

Los humedales son ecosistemas muy productivos y fuente importante de diversidad biológica, son esenciales para la subsistencia de una variedad de especies animales y vegetales, de manera permanente o esporádica, los que son, a su vez, importantes depósitos de material genético (MINAM 2015: 6). Dentro de los cinco tipos de humedales naturales que define la Convención Ramsar, los 
LOS HUMEDALES EN LA EVALUACIÓN DE IMPACTO AMBIENTAL DEL PROPUESTO AEROPUERTO DE Chinchero / Carlos Antonio Martin Soria Dall’Orso

humedales ubicados en la Región Cusco están clasificados dentro de dos grandes sistemas: palustres (bofedales) y lacustres (lagunas, lagunillas).

Los humedales proveen servicios ecosistémicos (MINAM 2019: 13-14) de:

a) Provisión: forraje para ganado y fauna silvestre ${ }^{4}$; la turba es abono para viveros y fuente de combustible, plantas medicinales, agua.

b) Regulación: almacena carbono en forma de turba; protege de la erosión de la turba y el suelo, pues reduce la velocidad del agua moviéndose dentro del bofedal; purifica agua; almacena agua; regulación hídrica; regulación del clima local y global.

c) Soporte: refugio de fauna silvestre y biodiversidad, mantenimiento del ciclo de nutrientes, la turba mantiene el bofedal.

d) Cultura: belleza escénica y paisajística; áreas de recreación y turismo.

Por estos servicios, los humedales tienen una relevancia ambiental y social, pues:

[...] las poblaciones cercanas los han utilizado desde siempre no solo como fuente de agua, sino para la extracción de productos necesarios para su vida diaria, como son materiales de construcción (junco y totora) o alimentos (pescado, mariscos, frutos, etc.). (MINAM 2015: 6)

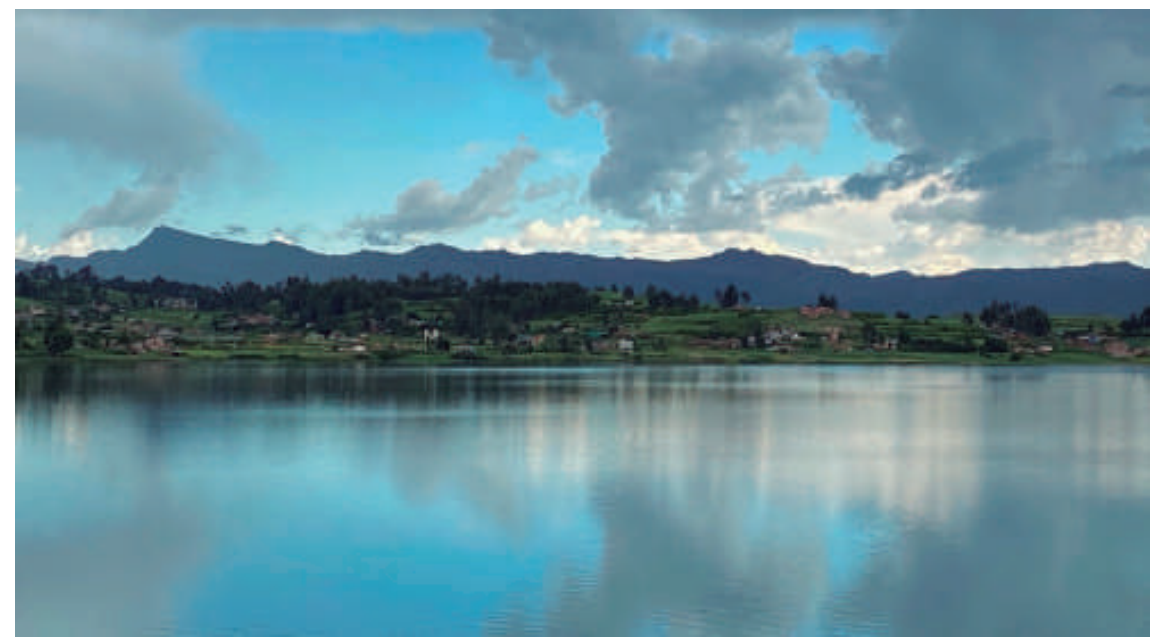

Imagen 3. Laguna Piuray. Fuente: Soria (2020).

\footnotetext{
4 «[...] además existen numerosas hierbas silvestres con propiedades medicinales y una diversidad de fauna silvestre que se congrega con mayor frecuencia alrededor de las lagunas de Piuray y Huaypo, así como en las diferentes lagunillas del distrito». (Municipalidad Distrital de Chinchero 2016: 46)
} 
REVISTA KAWSAYPACHA: SOCIEDAD Y MEDIO AMBIENTE $N^{\circ} 5$ (2020)

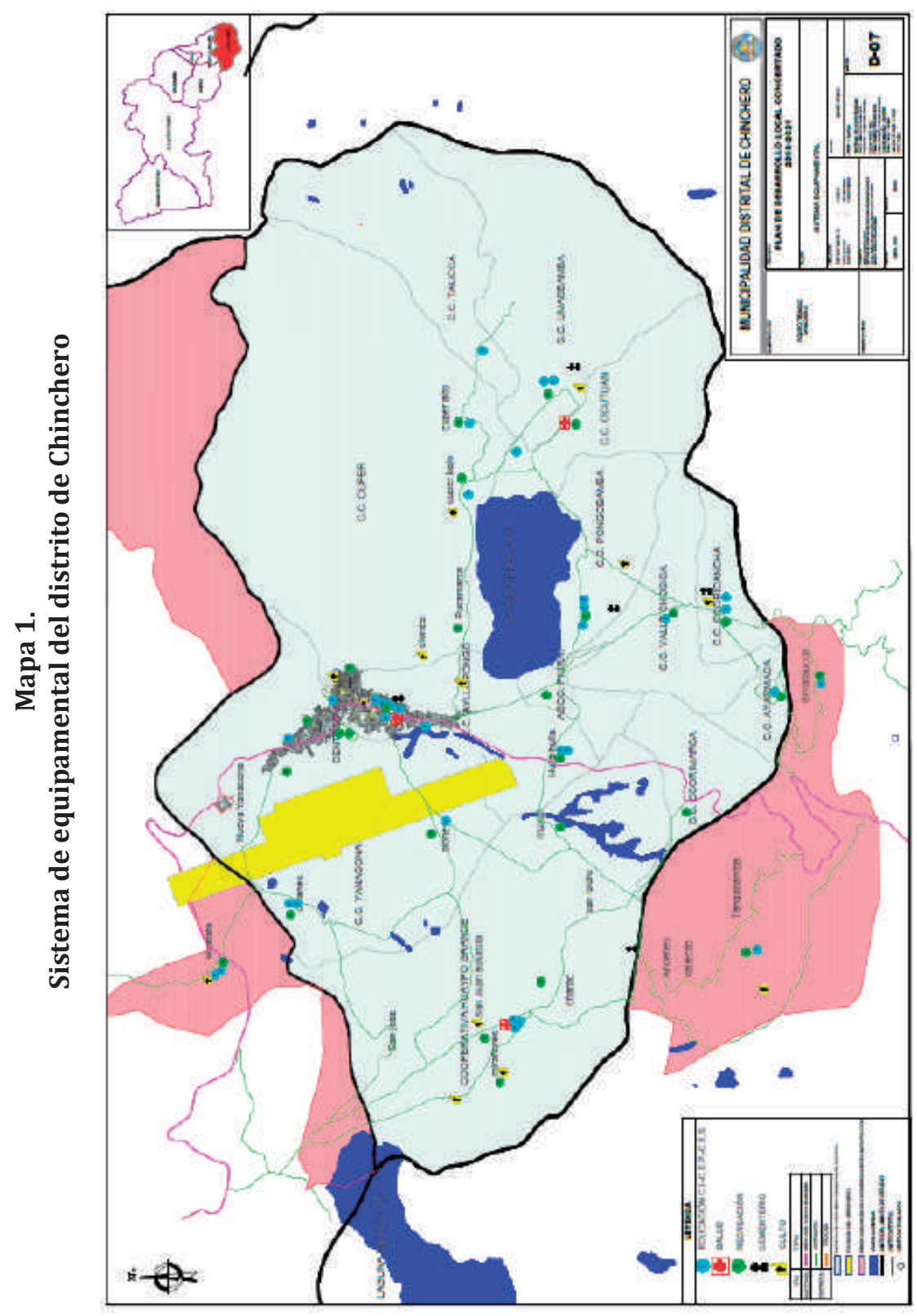

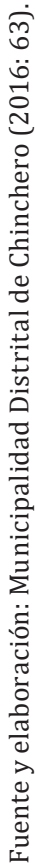


La mayoría de los humedales son alimentados por alguna fuente subterránea de agua, además de la precipitación. El aporte de agua que recibe depende de su posición en la cuenca y determina si es permanente o estacional. Cuando la napa freática está cerca de la superficie, saturando el suelo, la acumulación de turba se hace más lenta pues reduce la disponibilidad de oxígeno en el suelo y favorece la descomposición anaeróbica. En los humedales altoandinos, como los de Chinchero, se reduce la velocidad de cualquier reacción química y la disponibilidad de oxígeno en la atmósfera (MINAM 2019: 12).

Las mayores amenazas a los bofedales son los cambios en la provisión y cantidad de agua que alimenta el bofedal. Uno de los indicadores de alteraciones en el paisaje, a considerar para evaluar la salud del humedal, es la conectividad hidrológica. Es decir, determinar si hay infraestructura u otras evidencias de alteraciones en el curso del agua que afecten la calidad y cantidad de agua que debería llegar directamente al bofedal (MINAM 2019: 15).

La imagen 4 muestra la distribución de buena parte de los humedales sobre el espacio distrital.

Chinchero tiene flujos y recursos hidrológicos importantes confinados en su cuenca de $18,54 \mathrm{~km}^{2}$, distribuidos hidrogeológicamente en tres tipos de acuíferos (ALG-Europraxis 2013: 99):

a) Las 874,4 ha (47,7\% del área total de la microcuenca) de acuitardos, que por sus características litológicas son áreas de infiltración local de aguas subterráneas que se manifiestan en torno a la microcuenca.

b) Las 437,1 ha $(23,6 \%$ de la superficie total) de acuíferos porosos no consolidados que forman el colchón de mayor recepción de aguas pluviales, con una napa freática superficial que almacena agua en sus niveles confinados.

c) Las 95,5 ha (5,2\% del total) de acuíferos kársticos donde se presentan manantes locales.

El sistema hídrico de Chinchero tiene hoy:

a) La laguna Huaypo de 1,5 km por $800 \mathrm{~m}$ de ancho que aporta al sistema de riego Sambor-Huaypo y permite regar tierras de la provincia de Anta.

b) La laguna Piuray, que provee 239,08 litros por segundo, y sus manantes asociados (Urmana I, Urmana II, Tintinpucjio, Huayna Kor Kor, Ñahuinpucjio, Cuncunya y Fortaleza Nueva) que aportan otros 52,67 litros por segundo para el abastecimiento de agua potable de 125.488 cusqueños de los barrios del Centro Histórico, Santiago y San 
Sebastián, constituyendo el 38\% del agua potable para la ciudad de Cusco. Esta laguna alberga 39 especies de flora y 13 especies de fauna (EPS Sedacusco 2014: 37, 69, 114-115, 134; Municipalidad Distrital de Chinchero 2016: 46-47).

Hasta antes del inicio de las obras de remoción del 10\% de tierras del polígono del propuesto aeropuerto de Chinchero, el sistema hídrico de Chinchero también incluía:

c) Chaquel cocha, que era una laguna temporal de verano dentro de las tierras de la comunidad campesina Yanacona. Una vez vendidas estas tierras al MTC, se drenó Chaquel cocha y se cubrió con tierra.

d) El humedal Ponqolay, que era un humedal por afloramiento de aguas que era utilizado para regar, a través de acueductos ancestrales, cultivos de papa, quinua y kiwicha en las comunidades Yanacona y Huila Huila, y era hábitat de patos y otras aves (Cárdenas 2019a). El uso de las aguas del humedal Pongolay fue cedido a Altesa, la empresa a cargo de realizar el movimiento del $10 \%$ de tierras del polígono, mediante una resolución de la Autoridad Nacional del Agua (ANA) en un procedimiento administrativo que no consideró ni la consulta previa, ni los derechos ancestrales de estas poblaciones.

La Junta de Regantes del sector Chacapunku Pongolay (2019), que realizaba faenas de limpieza y mantenimiento de zanjas y acueductos para regar todos los años en el mes de setiembre, denunció en setiembre de 2019 que algunos camiones cisterna sobreexplotaban el humedal utilizando esas aguas en los trabajos de remoción del 10\% de tierras del polígono del futuro aeropuerto de Chinchero y afectando la siembra de productos agrícolas y la estabilidad. En setiembre de 2019, cuando se hizo la denuncia, el humedal se encontraba en proceso de eutrofización severa, mientras que reducía el nivel de su espejo de agua. El deterioro de la calidad del agua no permitió regar cultivos e incluso causó la muerte de patos silvestres. Esta laguna era fuente de recarga hídrica para la laguna de Piuray. Este humedal fue drenado, secado y cubierto de tierra (Cárdenas 2019b).

\section{El Estudio de Impacto Ambiental}

El Estudio de Impacto Ambiental (EIA) del aeropuerto de Chinchero fue elaborado por ACON Ambiental Consultores S.A.C. para Kuntur Wasi, y presentado por esta para revisión y aprobación del Ministerio de Transportes y Comunicaciones (MTC) en 2015. EL EIA identifica 4 
microcuencas en el área de influencia (ACON \& Kuntur Wasi 2015: 355), al igual que lo hizo el Estudio de Preinversión (ALG-Europraxis 2013: 787), es decir, la microcuenca de la laguna de Huaypo $\left(38,1 \mathrm{~km}^{2}\right)$; la microcuenca del río Corimarca $\left(81,7 \mathrm{~km}^{2}\right)$ que incluye la laguna de Piuray; la microcuenca de la quebrada de Urquillos $\left(44,4 \mathrm{~km}^{2}\right)$; y la microcuenca de la quebrada de Marcuyohuayjo $\left(13,5 \mathrm{~km}^{2}\right)$. Sin embargo, el EIA no inventarió todas las fuentes ni todos los usos del agua en las unidades hidrográficas del área de influencia directa e indirecta del proyecto y no precisó qué ocurrirá con estas durante y después de la construcción, ni tampoco establece medidas de manejo ambiental para reducir los impactos. Al hacer el inventario de fuentes de agua (ACON 2015: 356; ALG-Europraxis 2013: 788), de estructuras hidráulicas y de fuentes de agua natural (ACON \& Kuntur Wasi 2015: 356), únicamente se hizo el balance hídrico de la cuenca del río Corimarca (ACON \& Kuntur Wasi 2015: 393).

En cuanto al factor afectado «agua superficial», el EIA del aeropuerto de Chinchero (ACON \& Kuntur Wasi 2015: 620-621) lo caracteriza como afectado por efluentes líquidos, residuos líquidos y drenaje durante las actividades preliminares, movimientos de tierras, pavimentación, drenaje, edificaciones y operaciones con potenciales impactos de deterioro de la calidad del agua calificados con -9,5 en la escala de medición de los impactos ambientales (IAM) que presenta el propio EIA y lo clasifica como compatible, mientras que los de la modificación del patrón de drenaje, calificados con 0 , los clasifica como compatible.

Al referirse a las aguas subterráneas, el EIA identifica el acuífero en la zona (ACON \& Kuntur Wasi 2015: 407) y la napa freática (ACON \& Kuntur Wasi 2015: 409) con el balance hídrico correspondiente. En cuanto al factor afectado «agua subterránea», el EIA del aeropuerto de Chinchero (ACON \& Kuntur Wasi 2015: 621-622) lo caracteriza como calidad del agua subterránea y califica los impactos potenciales del deterioro de la calidad del agua con $-5,5$ y lo clasifica como compatible. En cuanto al agotamiento de reservas de agua, este es calificado con -11 IAM y clasificado como compatible. Se afirma que se agotará la reserva de agua renovable según el estudio hidrogeológico, la que se recuperará durante la época de lluvias. En relación con los impactos del potencial deterioro de la calidad del agua durante la etapa de operación del aeropuerto de Chinchero, el EIA los clasifica como IAM -7 (ACON \& Kuntur Wasi 2015: 633). El EIA del aeropuerto de Chinchero dice que solo se utilizará la reserva de agua renovable y no la permanente, y señala que el índice de calidad ambiental sobre la calidad de agua subterránea resulta compatible porque las probabilidades de que se dé algún impacto son escasas, pero sin dar muestra alguna de su análisis de riesgo, la serie de datos usada para tal afirmación o la evidencia provista por el seguimiento de este factor a lo largo del tiempo. 


\section{Análisis}

El Gobierno Regional del Cusco establece en la zonificación ecológica económica desarrollada para el departamento, que para el caso de las zonas de cultivo en limpio en sierra de calidad agrológica media, como es el caso de la pampa de Chinchero: «En estos suelos no es recomendable la realización de actividades de forestación ni reforestación, igualmente se debe prohibir el cambio de uso con fines de instalación de infraestructura urbano industrial» (Gobierno Regional del Cusco 2005: 117 y 118) ${ }^{5}$ debido a que es una parte del 0,6\% de la superficie departamental de tierras de pendiente plana o llana, que corresponde a fondos de valle aluvial montañoso y llanura de valle aluvial, con suelos profundos, que permiten araduras continuas y la utilización de maquinaria agrícola. En estas zonas de cultivo en limpio en sierra de calidad agrológica media, se utiliza el suelo de manera intensiva, con instalación de cultivos, bajo sistemas de riego tradicional o tecnificado, con cultivos de maíz, leguminosas, hortalizas, papa, pastos, alfalfa, entre otros. Se trata de suelos con buenas características edáficas, fertilidad natural media, pH moderadamente ácido a neutro, de textura media a moderadamente fina, moderadamente profundos, de buen drenaje, buena retención de humedad, que facilita el desarrollo y manejo de los cultivos instalados (2005: 117 y 118).

El EIA (ACON \& Kuntur Wasi 2015: 358) señala que en el polígono del propuesto aeropuerto de Chinchero no se ha identificado manantiales ni bofedales de importancia dentro del área de influencia directa. Sin embargo, el Estudio de Preinversión (ALG-Europraxis 2013: 98-99) afirma que «el agua (en la zona del proyecto) proviene de los manantiales que afloran en la parte media y baja de la microcuenca conformando bofedales importantes». Esta clara contradicción es una vulneración de los principios de veracidad, buena fe y verdad material (artículo IV del Texto Único Ordenado de la Ley del Procedimiento Administrativo General) aplicables a cualquier procedimiento administrativo, del contenido de los instrumentos de gestión ambiental (artículo 10 de la ley 27446, Ley del SEIA) y con consecuencias en el ámbito administrativo y penal.

El EIA no evalúa el potencial impacto ambiental sobre las lagunas Piuray y Huaipo, los humedales Chápar y Chacapunku Pongolay, los humedales estacionales como Chaquel cocha, o los aproximadamente 50 manantiales y varios canales incas y coloniales. La variación de los niveles de agua de la laguna de Piuray (a 3.645 m s. n. m.) registrada en la última década y el deterioro de la calidad de agua por efecto de la contaminación ambiental en la cuenca son amenazas a la seguridad del agua potable para Cusco (EPS Sedacusco 2014: 62). 
Los humedales de Chinchero juegan un papel muy importante en el mantenimiento y la regulación hídrica de la cuenca. En 2018, la EPS Sedacusco financió S/ 701.679 para el mejoramiento y recuperación de los servicios ecosistémicos de la laguna de Piuray, con la instalación de especies forestales en la comunidad campesina de Pongobamba para la regulación de la microcuenca de Piuray-Corimarca en el distrito de Chinchero (código SNIP 2378896) (EPS Sedacusco 2020). La Municipalidad Distrital de Chinchero, en su Plan de Desarrollo Concertado al 2021, considera que «el recurso hídrico se encuentra en riesgo de disminución y pérdida, por lo que, concordante con su aprovechamiento el consumo para agua potable y riego requiere ser conservado» (2016: 47 y 69). Por ello, es imperativo que una intervención de la escala del propuesto aeropuerto de Chinchero incorpore en su plan de ingeniería aspectos como operación y mitigación sobre estas fuentes de agua que el Estado ve como de valor estratégico.

La afectación y, en este caso, la pérdida de humedales debe ser valorada y proceder a la compensación, reestableciendo nuevos humedales o ampliando otros humedales que compensen el servicio perdido, de acuerdo con las normas de la legislación nacional.

Además de los impactos sobre el humedal como ecosistema, se suma el impacto socioeconómico sobre la población local, pues el EIA del propuesto aeropuerto de Chinchero no hace referencia al sistema hídrico compuesto por los humedales que abastecen de agua a Chinchero, las comunidades, la Ciudad del Cusco y los distritos de Urubamba y Anta. La riqueza natural de este sistema hídrico sostiene a la población humana, la flora y la fauna altoandina de Chinchero y sus comunidades, por tanto, también a la agrobiodiversidad ahí presente. Sin embargo, el EIA del propuesto aeropuerto de Chinchero no ha descrito debidamente los potenciales impactos sobre este sistema, no ha considerado los antecedentes de la zona de influencia, tampoco ha identificado y caracterizado las implicaciones e impactos ambientales negativos, ni ha definido metas ambientales como parte de una estrategia de manejo ambiental para este impacto, según lo ordenan los literales a), b) y c) del artículo 10 de la ley 27446, Ley del SEIA.

La Municipalidad Distrital de Chinchero reporta que en 2015 la tasa de desnutrición crónica en niños menores de 5 años fue de $17 \%$, mostrando déficit de la talla, «peso insuficiente para la talla por la baja ingestión de alimentos y consumo de agua de mala calidad, lo que muestra la alta vulnerabilidad y la inseguridad alimentaria y nutricional» ${ }^{6}$ (Municipalidad Distrital de Chinchero 2016: 10). Con respecto a la anemia en niños menores de 3 años, en 2015 fue del 74,5\% (2016: 10). Este impacto se atribuye localmente, en parte, al hecho de que desde que inició el proceso de adquisición de tierras 
REVISTA KAWSAYPACHA: SOCIEDAD Y MEDIO AMBIENTE N 5 (2020)

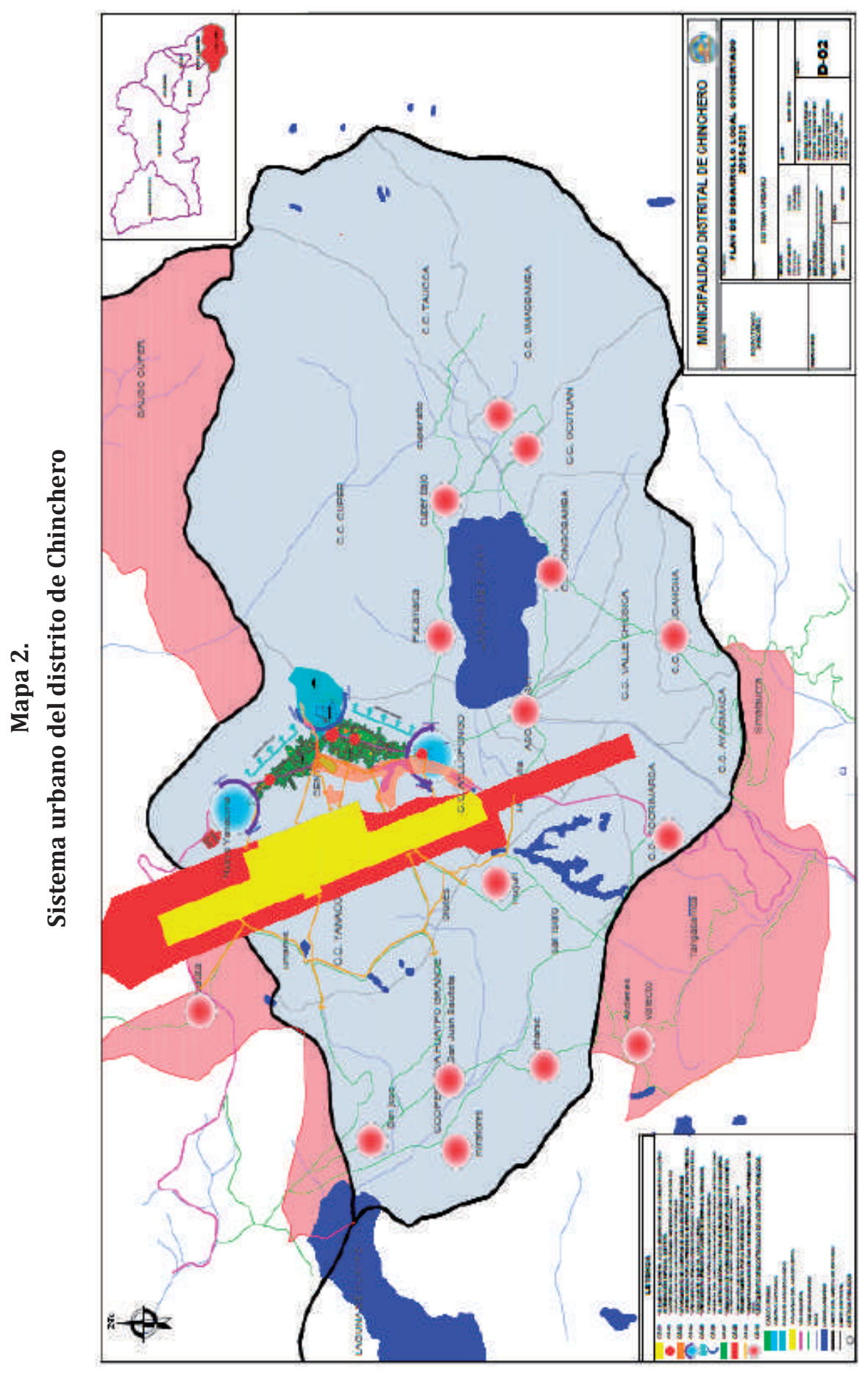

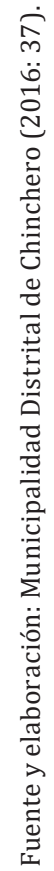


del polígono del futuro aeropuerto de Chinchero, esas tierras dejaron de producir productos agrícolas anteriormente destinados al consumo local y al pago del servicio al templo.

En la imagen 5 se muestra la ubicación del polígono del propuesto aeropuerto de Chinchero. Dicho polígono producirá un fraccionamiento del distrito en dos sectores, oriente y occidente, cortar el acceso al sector oeste del distrito afecta a algunas comunidades campesinas. Así también, la implantación de un polígono de 500 ha asfaltadas, de las 9.457 ha que tiene el distrito, producirá la desarticulación espacial de la conectividad hídrica del sistema de humedales de Chinchero. La estabilización del terreno afecta al sistema de humedales, especialmente los manantiales que quedarán cubiertos de tierra, como ya ocurrió en el caso de Chaquel cocha y Pongolay. Las tierras de producción agropecuaria dejaron ya de usarse para tal fin desde que fueron adquiridas por el Ministerio de Transportes y Comunicaciones. A lo que en el futuro se agregará la construcción de carreteras para acceso al aeropuerto. Por todas estas razones, el EIA vulnera los principios de sostenibilidad, de valoración del agua y precautorio de la Ley de Recursos Hídricos, ley 29338/2009, así como del sistema de evaluación ambiental, como veremos más adelante.

La identificación, caracterización, dimensionamiento y valoración de los impactos ambientales de la construcción y operación del propuesto aeropuerto de Chinchero es pobre, parcial, deficiente y limita la formulación del Plan de Manejo Ambiental. Como se puede ver en la imagen 6, la tabla de valoración de criterios de los rangos de valoración de los impactos negativos y positivos carece de valores entre $1 \mathrm{y} 7$, y el primer rango que presenta va entre 8 y 14, tanto en la escala positiva como negativa.

Tabla 1.

Clasificación Rangos: Impactos Negativos (IN) Impactos Positivos (IP).

\begin{tabular}{|c|c|c|} 
& Rango del índice de impacto & Calificación \\
\hline \multirow{2}{*}{$\begin{array}{l}\text { Impactos } \\
\text { negativos }\end{array}$} & -24 a -23 & Crítico \\
\cline { 2 - 3 } & -22 a -20 & Severo \\
\hline & -19 a -15 & Moderado \\
\hline & -14 a -8 & Compatible \\
\hline \multirow{2}{*}{$\begin{array}{l}\text { Impactos } \\
\text { positivos }\end{array}$} & 8 a 14 & Calificación \\
\cline { 2 - 3 } & 15 a 19 & Bajo \\
\hline & 20 a 22 & Medio \\
\hline & 23 a 24 & Alto \\
\hline
\end{tabular}


Por otro lado, el ingeniero agrícola y especialista en gestión social del agua, Marco Zeisser (2018), caracteriza las deficiencias del EIA en el tema agua-humedales como:

1. Solo describe algunos impactos dentro del polígono del aeropuerto. Por ejemplo, sobre los residuos líquidos se propone la construcción de una planta de tratamiento de aguas dentro del aeropuerto, pero no considera el impacto sobre el área de influencia indirecta. Igualmente, se ofrece el monitoreo de las aguas superficiales y subterráneas y del agua de consumo dentro del polígono del aeropuerto, pero no evalúa estos factores en las áreas de influencia directa e indirecta.

2. Solo hace el balance hídrico de la microcuenca del río Corimarca y no de las microcuencas Huaypo, Marcuyohuayjo y Urquillos.

3. No registra, caracteriza o valora el aporte de los humedales, ni su contribución a los sistemas de agua potable.

4. No registra los sistemas de riego existentes.

5. Todos los índices de impacto ambiental son calificados entre $-5,5$ a -12 , y clasificados como compatibles indicando que la recuperación de las condiciones ambientales es inmediata tras el cese de la actividad, aun sin precisar las prácticas protectoras o correctoras.

6. Sobre el agua superficial, el EIA considera poco probable, y en todo caso manejable, la posibilidad de un accidente que afecte la calidad, por lo que califica como -9,5, sin dar evidencia de su análisis y proyección, minimizando los riesgos de contaminación, aun pese a la complejidad de las actividades en el periodo de construcción, más aún si tenemos en cuenta que estas actividades incluyen la operación de una planta de asfalto. Luego se evalúa que el agotamiento de las reservas es poco probable por baja demanda versus la disponibilidad de la reserva renovable, y se califica como -11 y compatible. Sobre el deterioro de la calidad del agua subterránea por derrames accidentales, recibe la misma evaluación que en la fase de construcción, pero IAM -7, y se clasifica como compatible. Sobre el bombeo de agua de la reserva renovable, calificada con -12 y clasificada como compatible, no se considera la demanda de usuarios diferentes al aeropuerto, por tanto, afirmar que «el agotamiento de la reserva es poco probable» (ACON \& Kuntur Wasi 2015: 621-622) no es un dato confiable. Es innegable que en todos estos casos se minimizan los riesgos de agotamiento sin dar evidencia del análisis que lleva a esa conclusión.

7. La valoración del impacto sobre el patrón de drenaje es errada. El EIA afirma que el drenaje natural podría verse afectado de manera temporal y lo clasifica con IAM 0. Sin embargo, la nivelación y compactación del terreno ondulado y la impermeabilización de centenares de hectáreas 
modifica el patrón de drenaje, el escurrimiento, la infiltración de las aguas, el comportamiento del acuífero y, en consecuencia, las actividades agropecuarias. Este es un impacto definitivo que no se recupera sin medidas correctoras.

8. No valora el impacto del proceso de urbanización en un periodo de al menos cuarenta años de operación sobre el sistema hídrico del distrito.

Además de estas limitaciones, el EIA tampoco considera el impacto del proceso de construcción de infraestructura lineal (carreteras y líneas de conducción eléctrica) sobre los cursos de agua subterráneos y superficiales que alimentan a los sistemas de humedales, en especial a los bofedales, pues el impacto es más difícil de observar. Las vías de acceso pueden afectar los cursos de agua que alimentan humedales. Estos dos factores aumentarán la impermeabilización y el fraccionamiento.

Si bien el EIA del propuesto aeropuerto de Chinchero no aborda el tema, el Plan de Desarrollo Concertado al 2021 de la Municipalidad Distrital de Chinchero (2016: 69) advierte que en el contexto del cambio climático «el recurso hídrico se encuentra en riesgo de disminución y pérdida, por lo que, concordante con su aprovechamiento el consumo para agua potable y riego requiere ser conservado». Más aún si tenemos en cuenta que la laguna de Piuray abastece de agua a la Ciudad del Cusco.

Los bofedales de Chinchero incluyen turberas, aquellos en los que la capa de materia orgánica es igual o mayor a $40 \mathrm{~cm}$ de profundidad, por tanto, son importantes sumideros de carbono orgánico en el suelo. Si se desecan, estas turberas emiten los gases de carbono atrapados en el suelo hacia la atmósfera, contribuyendo a elevar los niveles de $\mathrm{CO}_{2} \mathrm{y}$, por tanto, al calentamiento global.

Además de los bofedales naturales, también hay los que han sido construidos desde tiempos prehispánicos por medio de canales y otros medios que ampliaron zonas de formación natural (Flores Ochoa 1977, Lane 2009). La consultora Trashumantes (2012: 11), en un estudio elaborado por la arqueóloga Lucía Balbuena Cotlear, declaró el hallazgo de 7 fragmentos de cerámicos, 3 caminos inca y 13 rutas coloniales dentro de las, en ese entonces, 357 ha del proyecto, confirmando así que estas tierras forman parte de un Paisaje Cultural Arqueológico y que los humedales juegan un papel fundamental importante en la estructura cultural y de producción agrícola de la zona. Un estudio arqueológico debió dilucidar este extremo, lamentablemente el EIA del propuesto aeropuerto de Chinchero no aborda este factor.

El cambio climático puede traer riesgo de precipitaciones extremas incrementando el riesgo de inundaciones. Las condiciones geomorfológicas de Chinchero facilitan el rápido incremento del caudal de los cursos de agua, 
lagunas y humedales, especialmente en época de lluvias, afectando el área del propuesto aeropuerto de Chinchero, «en donde el nivel freático se encuentra cercano a la superficie» (Municipalidad Distrital de Chinchero 2016: 48). Al oeste de Chinchero, los humedales son un peligro muy alto para el estadio y algunas viviendas. Si bien las tierras del propuesto aeropuerto de Chinchero serán niveladas, la provisión de aguas continuará manando desde la laguna Huaypo y causando inundaciones. Al lado este, las laderas tienen peligro de derrumbe. En la zona rural, las áreas cercanas a los cuerpos de agua serán

[...] afectadas por inundación si se presentan lluvias extraordinarias, en ese sentido, se observa que el proceso de expansión y densificación urbana y rural se encuentra expuesta y vulnerable a peligros que ponen en riesgo la vida, los activos físicos, entre otros, de la población. (2016: 48)

El cambio climático puede modificar el comportamiento de la temperatura en Chinchero con eventos de frío extremo (friaje) bajo los $0{ }^{\circ} \mathrm{C}$ (Municipalidad Distrital de Chinchero 2016: 49-50). En décadas pasadas, las granizadas se presentaban en las zonas altas, en años recientes se presentan en diferentes sectores y comunidades del distrito (2016: 49-50). Esto ha favorecido el incremento de la intensidad de las granizadas y es otro elemento que el EIA del aeropuerto de Chinchero debería caracterizar, dimensionar y proponer acciones de mitigación en el Plan de Manejo Ambiental, debido al uso de químicos en el control del enfriamiento de aeronaves y congelación de la pista. Esos químicos deben recuperarse para evitar que escurran al sistema hídrico natural.

Barbier, Ackerman y Knowler (1997: viii) resaltan que la «noción de que los humedales son tierras inservibles ha dado lugar a la desecación o conversión de muchos de ellos para dedicarlos a la agricultura intensiva, la acuicultura, la industria o la vivienda o para promover la salud pública». Esta noción está implícita en el EIA del aeropuerto de Chinchero cuando no los aborda y no contiene ninguna medida de manejo ambiental para un proyecto que afecta irremediablemente a este sistema de humedales.

La afectación de los humedales de Chinchero vulnera compromisos del Gobierno del Perú con el derecho internacional, como los artículos $3^{7}$ y $4^{8}$ de la Convención Ramsar, que protegen todos los humedales de un país, no solo los declarados como humedales Ramsar. Además, la Convención Ramsar

\footnotetext{
Artículo 3, párrafo 1. Las Partes Contratantes deberán elaborar y aplicar su planificación de forma que favorezca la conservación de los humedales incluidos en la Lista y, en la medida de lo posible, el uso racional de los humedales de su territorio. (Las cursivas son mías.)

8 Artículo 4, párrafo 1. Cada Parte Contratante fomentará la conservación de los humedales y de las aves acuáticas creando reservas naturales en aquéllos, estén o no incluidos en la Lista, y tomará las medidas adecuadas para su custodia.
} 
LOS HUMEDALES EN LA EVALUACión DE IMPACTO AMBIENTAL DEL PROPUESTO AEROPUERTO DE Chinchero / Carlos Antonio Martin Soria Dall'Orso

y sus partes contratantes (Secretaría de la Convención de Ramsar 2010: 41) recomiendan:

Evitar nuevas pérdidas de los humedales [...] Reconocer y alentar la función y los esfuerzos de las comunidades locales» [y] Coordinar y racionalizar los programas gubernamentales a fin de reducir al mínimo sus efectos perjudiciales en los humedales y alentar en cambio la conservación de estos.

En el ámbito de la legislación nacional, se vulnera la Ley General del Ambiente, ley 28611/2005, que en referencia al agua continental ordena que:

El Estado promueve y controla el aprovechamiento sostenible de las aguas continentales a través de la gestión integrada del recurso hídrico, previniendo la afectación de su calidad ambiental y de las condiciones naturales de su entorno, como parte del ecosistema donde se encuentran; regula su asignación en función de objetivos sociales, ambientales y económicos; y promueve la inversión y participación del sector privado en el aprovechamiento sostenible del recurso. (Artículo 90) ${ }^{9}$

Igualmente, esta ley establece que los ecosistemas frágiles «comprenden, entre otros, [...] pantanos, bofedales, [...], humedales, lagunas altoandinas» (artículo 99, párrafo 2), y dispone que «las autoridades adoptan medidas de protección especial para los ecosistemas frágiles, tomando en cuenta sus características y recursos singulares; y su relación con condiciones climáticas especiales y con los desastres naturales» (artículo 99, párrafo 1) y que «El Estado reconoce la importancia de los humedales como hábitat de especies de flora y fauna, en particular de aves migratorias, priorizando su conservación en relación con otros usos» (artículo 99, párrafo 3).

El polígono de 500 ha del propuesto aeropuerto de Chinchero afectará a especies sensibles y especializadas que quedarán restringidas a los fragmentos restantes de paisaje natural no perturbado. Maldonado Fonken $(2014: 2,10)$ señala que en otros casos similares «En este contexto, los bofedales se han convertido en centros de biodiversidad y un recurso importante para las personas». Concordante con ello, el Reglamento de Zonificación Ecológica Económica Peruana (decreto supremo 087-2004-PCM, artículo 9) clasifica a los bofedales como áreas de protección y conservación ecológica.

9 El énfasis en cursivas es del autor. 


\section{Conclusión}

Las deficiencias, errores, falsedades y carencias de la línea de base, de la identificación y evaluación de los impactos ambientales y en la estrategia de manejo ambiental del EIA del propuesto Aeropuerto de Chinchero, en materia de aguas superficiales y subterráneas, incluyendo los humedales, vulneran la Constitución Política del Perú, la Ley General del Ambiente, la Ley de Recursos Hídricos, la Ley del Sistema Nacional de Evaluación de Impacto Ambiental (SEIA), la Ley del Sistema Nacional de Evaluación y Fiscalización Ambiental, con consecuencias administrativas y en algún caso incluso penal.

OEFA conoce casos de evaluaciones ambientales con inadecuada línea de base, sea que esta no evalúa «componentes esenciales (manantiales, lagunas)» o porque no se realizó «estudios técnicos para determinar la calidad ambiental (estudios hidrológicos, estudios del suelo, entre otros). Este tipo de deficiencias generan dificultades o barreras para un adecuado ejercicio la fiscalización ambiental» (OEFA 2016: 148), que impide comparaciones sobre la calidad del ambiente, antes, durante y después del desarrollo del proyecto y dificulta determinar responsabilidades administrativas. Obviamente, que sea el administrado quien determine la línea base y provea los datos con los que luego va a ser fiscalizado, no es un mecanismo eficiente y favorece estos incumplimientos. La corrección de este problema vía actualización del EIA no es del todo certera y eficaz, en tanto ocurre post facto, por lo menos respecto del $10 \%$ de tierras que ya se han movido y nivelado conteniendo humedales ya perdidos.

Los tres niveles de autoridades gubernamentales en materia de supervisión, fiscalización y sanción ambiental ejercen funciones en el SEIA y son responsables de efectuar las funciones de supervisión, fiscalización y sanción, relacionadas con el cumplimiento de las obligaciones asumidas en el estudio ambiental aprobado y el marco normativo ambiental vigente, desde el inicio de las obras para la ejecución del proyecto (artículo 77 del decreto supremo 019-2009-MINAM). Lamentablemente, OEFA ha encontrado, en materia de residuos sólidos y de minería, que los Gobiernos regionales no cumplen con «realizar acciones de monitoreo y evaluación ambiental, o iniciar procedimientos administrativos sancionadores ante una presunta infracción ambiental» (Gómez \& Granados 2015: 94).

Tanto el Ministerio de Transportes y Comunicaciones como el Ministerio de Ambiente deberían realizar acciones de supervisión y fiscalización de las obligaciones establecidas en el EIA del aeropuerto de Chinchero, para determinar si los impactos ambientales negativos generados difieren de manera significativa de aquellos declarados durante el proceso de certificación ambiental. Si así fuera, la autoridad en el ámbito del SEIA debería requerir al titular la adopción de medidas correctivas o de manejo 
ambiental que resulten necesarias para mitigar y controlar sus efectos, sin perjuicio de requerir la actualización del estudio ambiental, en el plazo y condiciones que indique de acuerdo con la legislación vigente. Esta condición no exceptúa la eventual paralización de operaciones o la aplicación de otras sanciones que pudieran corresponder (artículo 78 del decreto supremo 019 2009 MINAM).

El Ministerio de Transportes y Comunicaciones debe solicitar la opinión técnica del Ministerio de Cultura, así como el Certificado de Inexistencia de Restos Arqueológicos, y adoptar medidas correctivas y de manejo ambiental para el propuesto aeropuerto de Chinchero y disponer la actualización del estudio ambiental en función de la existencia de acueductos ancestrales de uso presente, por lo que incluso hoy puede ordenar la paralización de actividades y la aplicación de sanciones.

El EIA del aeropuerto de Chinchero debió considerar los aspectos vinculados al cambio climático, así como otros no reseñados aquí pero que incluyen cambios en las épocas de fructificación y floración de las especies de flora, épocas de reproducción de las especies de fauna, entre otras. No hacerlo es otra seria omisión que afecta su utilidad como instrumento de gestión ambiental y pone en riesgo a la biodiversidad y la población. Estos otros factores se ven afectados por la afectación del sistema hídrico de Chinchero y la reducción de los humedales, ya causada por la remoción del $10 \%$ de tierras del polígono del futuro aeropuerto de Chinchero, pero que será aún mayor si el proyecto se efectiviza sobre el 90\% restante del polígono.

Por todos los aspectos revisados, el EIA del aeropuerto de Chinchero es deficiente en el análisis cuantitativo de los elementos que presenta, más aún en el análisis cualitativo de sus impactos sobre los humedales, con consecuencias sobre el medio natural, social y económico. En estas condiciones, el EIA no ofrece un análisis confiable de los impactos que generará el aeropuerto de Chinchero sobre el recurso agua y los humedales. De otro lado, el titular del proyecto no está sujeto al cumplimiento de compromisos ambientales en materia de humedales.

\section{Referencias}

ACON \& Kuntur Wasi (31 de mayo de 2015). Estudio de Impacto Ambiental Detallado del Nuevo Aeropuerto Internacional de Chinchero Cusco - Mejoramiento del Servicio Aeroportuario en la Región Cusco mediante el Nuevo Aeropuerto Internacional de Chinchero Cusco. Informe Final. Consorcio Kuntur Wasi.

ALG-Europraxis (3 de mayo de 2013). Estudios de Preinversión a nivel de perfil y factibilidad del Proyecto. Informe № 5.A y Anexos (Tomo I). Estudio de 
Preinversión a Nivel de Perfil. Versión final. Barcelona/Lima: ProInversión. https://www.academia.edu/30219791/Perfil_Aeropuerto_Chinchero

Barbier, E. B., Ackerman, M. C. y Knowler, D. (1997). Valoración económica de los humedales. Guía para decisores y planificadores. Oficina de la Convención de Ramsar, Gland, Suiza.

Capital Press (5 de septiembre de 2017). Northwest farmland values continue to increase. Capital Press. https://www.capitalpress.com/state/idaho/ northwest-farmland-values-continue-to-increase/article_15a64acec343-553b-8351-8a9be7cef942.html

Cárdenas, C. (2 de julio de 2019 a). Cusco: Aeropuerto de Chinchero avanza sobre caminos Inca y coloniales. Ojo Público, edición regional. https:// ojo-publico.com/1263/cusco-aeropuerto-de-chinchero-avanza-sobrecaminos-inca

Cárdenas, C. (14 de octubre de 2019 b). Aeropuerto de Chinchero no consideró impacto en cuencas que abastecen de agua a Cusco. Ojo Público, edición regional. https://ojo-publico.com/1435/aeropuerto-de-chinchero-noconsidero-impacto-en-lagunas-de-cusco

EPS Sedacusco (2014). Estudio de Factibilidad de Inversión Pública. Renovación Línea de Conducción Piuray. Código SNIP 113260. Enero. Cusco: EPS SEDACUSCO SA.

EPS Sedacusco 2020. Proyectos de Inversión. Recuperado el 18 de abril de 2020. https://www.sedacusco.com/transparencia/proyectos-de-inversion/

Flores Ochoa, J. A. (1977). Pastores de Puna. Lima, IEP.

Gobierno Regional del Cusco (2005). Zonificación Ecológica Económica Departamento del Cusco. Cusco: Dirección de Estudios y Proyectos de Gestión Ambiental. Cusco.

Gómez. H. y Granados, M. (2015). El macroproceso de la fiscalización ambiental. Revista de Derecho Administrativo, (15): 75-95.

Junta de regantes del sector Chacapunku Pongolay (2019). Humedales, lagunas y fauna silvestre muriendo iiiChinchero muriendo!!! Ojo al Agua. https:// ojoalagua.com/2019/09/06/humedales-lagunas-y-fauna-silvestremuriendo-chinchero-muriendo/

Lane, K. (2009). Engineered highlands: the social organization of water in the ancient north-central Andes (AD 1000-1480). World Archaeology, 41(1): 169-190. https://doi.org/10.1080/00438240802655245

La República (23 de diciembre.2017). Cusco: Gobierno pagará más de S/ 14 mllns por terrenos para Chinchero.

Maldonado Fonkén, M. S. (2014). Introducción a los bofedales de la región Altoandina Peruana. Mires and Peat, (15: art. 05). http://mires-and-peat. net/media/map15/map_15_05_Spanish.pdf.

Ministerio del Ambiente. (2015). Estrategia Nacional de Humedales. Lima, MINAM. 
LOS HUMEDALES EN LA EVALUACión DE IMPACTO AMBIENTAL DEL PROPUESTO AEROPUERTO DE Chinchero / Carlos Antonio Martin Soria Dall’Orso

Ministerio del Ambiente. (2019). Guía de Evaluación del Estado del Ecosistema de Bofedal. Lima, MINAM.

Municipalidad Distrital de Chinchero. (2016). Plan de Desarrollo Concertado al 2021. Chinchero: Municipalidad Distrital de Chinchero.

Novoa Arévalo, S. (2003). «Aeropuerto Internacional del Cuzco». ChincheroUrubamba (tesis de grado). UPC-Facultad de Arquitectura, Lima.

OEFA (2016). La vinculación y la retroalimentación entre la certificación y la fiscalización ambiental. Lima: OEFA.

Secretaría de la Convención de Ramsar. (2010). Políticas Nacionales de Humedales. Elaboración y aplicación de Políticas Nacionales de Humedales. Manuales Ramsar para el uso racional de los humedales, 4ํㅡㄹ edición, vol. 2. Secretaría de la Convención de Ramsar, Gland (Suiza).

Dodds, A. (28 de febrero de 2018). What does 2018 hold for the farmland market? Stags. https://www.stags.co.uk/articles/what-does-2018-hold-for-thefarmland-market

Trashumantes (2012). Plan de Monitoreo Arqueológico para el Aeropuerto Internacional de Chinchero. Departamento de Cusco. Entregable 4. Contrato 011-2011-Proinversion.

Zeisser, M. (abril de 2018). Estudio de impacto ambiental. Lagunas e insuficiencias. Revista del Aero Club del Perú : 49-56.

\section{Imágenes}

Imagen 1. Soria, D. A. M. (2020). Vista del palacio del Inca Túpac Yupanqui en Chinchero. Archivo personal.

Imagen 2. Soria, D. A. M. (2020). Alpacas, cultivos y nevado en el paisaje de Chinchero. Archivo personal.

Imagen 3. Soria, D. A. M. (2020). Laguna Piuray. Archivo personal.

Mapa 1. Municipalidad Distrital de Chinchero (2016). Mapa $\mathrm{N}^{\circ}$ 08. Sistema equipamental del distrito de Chinchero. [Mapa]. En Plan de Desarrollo Concertado al 2021 (p. 63). Chinchero: Municipalidad Distrital de Chinchero.

Mapa 2. Municipalidad Distrital de Chinchero (2016). Mapa $\mathrm{N}^{\circ} 03$ Sistema urbano del distrito de Chinchero. [Mapa]. En Plan de Desarrollo Concertado al 2021 (p. 37). Chinchero: Municipalidad Distrital de Chinchero. 